\title{
OPPORTUNISTIC AND PLAYBACK-SENSITIVE SCHEDULING FOR VIDEO STREAMING
}

\author{
Huda Adibah Mohd Ramli ${ }^{1}$ and Kumbesan Sandrasegaran ${ }^{2}$ \\ ${ }^{1}$ Department of Electrical and Computer Engineering, International Islamic University \\ Malaysia (IIUM), Kuala Lumpur, Malaysia \\ ${ }^{2}$ Faculty of Engineering and Information Technology, University of Technology, \\ Sydney, Australia
}

\begin{abstract}
Given the strict Quality of Service $(Q o S)$ requirements of video streaming, this paper proposes a novel solution for simultaneous streaming of multiple video sessions over a mobile cellular system. The proposed solution combines a buffer management strategy with a packet scheduling algorithm. The buffer management strategy selectively discards packets of a user from base station buffer whereas the packet scheduling algorithm schedules packets of a user according to its instantaneous channel quality, average throughput and playback buffer information. Simulation results demonstrate that the proposed solution is effective in providing a continuous video playback with good perceptual quality for more users. If at least a good perceptual quality is to be satisfied for all users (QoS constraint of video streaming), then the proposed solution improves the system capacity by $40 \%$ over a conventional packet scheduling algorithm.
\end{abstract}

\section{KEYWORDS}

Packet scheduling, video streaming, Long-Term Evolution, Quality of Service, Orthogonal Frequency Division Multiple Access.

\section{INTRODUCTION}

Long Term Evolution (LTE), which is now referred to as $3.9 \mathrm{G}$, is the latest commercially available Third Generation Partnership Project (3GPP) standard. The LTE is envisaged to provide a better quality of multimedia communications by providing higher data rates (50 Mbps in uplink and 100 Mbps in downlink), reduced latency and increased capacity and coverage. The LTE is a multi-carrier mobile cellular system. It uses Orthogonal Frequency Division Multiple Access (OFDMA) for downlink transmission. The bandwidth in the downlink LTE is divided into multiple equally spaced and mutually orthogonal sub-carriers. The minimum downlink LTE transmission unit that can be allocated to a user is referred to as a Resource Block (RB). An RB is made up of 12 sub-carriers of 1 ms duration [1].

Recent trends have shown an increase in popularity of video streaming application among mobile cellular users [2]. For transport over mobile cellular channels, a video stream is encoded (compressed) into frames of different properties (namely I, P and B frames) in order to reduce the bandwidth requirements [3]. There are multiple Group of Pictures (GoPs) within an encoded video stream. A GoP starts with an I frame and all frames prior to the subsequent I frame [4]. Each frame within a GoP has a different priority and is highly co-related. The I frame has the highest priority followed by the $\mathrm{P}$ and $\mathrm{B}$ frames. It should be noted that decoding of a $\mathrm{P}$ frame within a GoP is dependent upon an I frame within the same GoP and decoding of a B frame 
within a GoP is dependent upon an I and P frames within the same GoP. The loss of a higher priority frame within a GoP results in the loss of other dependent frames within the same GoP [5]. Even when the video stream is compressed, it still requires large bandwidth. Simultaneous transmissions of this bandwidth-hungry video streaming application is challenging as it may lead to a mobile cellular congestion if the expensive radio resources are not properly scheduled. Another challenge is that streaming of multiple video sessions simultaneously requires a continuous video playback at the highest perceptual quality at each user (Quality of Service, QoS, constraint of video streaming) [6].

Numerous studies have been discussed in the literature so as to address the stated challenges. For example, the authors in [7] developed a buffer management strategy that selectively discard packets of video users at the base station according to their priority and deadline. Note that packet is a segment of a video frame. This strategy ensures that the limited radio resources are efficiently used for transmission of packets that can be used for decoding and video playback and hence improving the perceptual quality experience at the users. Frequent interruptions during video playback may cause major annoyance to the video users. As such, a buffer management strategy that attempts to improve video playback continuity by minimizing the number of interruptions during video playback was developed in [8].

Besides the buffer management strategy, packet scheduling is another area of research interest when dealing with simultaneous transmission of multiple video users. Packet scheduling is responsible to efficiently select a user's packets for (re)transmission at a given time using an available radio resource so as to provide a satisfactory QoS, guarantee fairness and optimize system performance. Proportional Fair (PF) [9] is one of the well-known packet scheduling algorithms in the legacy single-carrier mobile cellular systems. This algorithm takes channel quality and average throughput of each user into consideration when making scheduling decision. In addition to that, a packet scheduling algorithm that delays transmission of the least important packets and allocates more radio resources for transmission to more important packets was developed in [10]. The algorithm was developed in order to avoid mobile cellular congestion and ensure a continuous video playback.

Other studies in the literature combined a buffer management strategy with packet scheduling algorithm. It should be noted that the following discussions refers the combination of a buffer management strategy with packet scheduling algorithm as a packet scheduling solution. To ensure timely arrival of video packets at a user, the authors in [11] developed a packet scheduling algorithm that schedules packets of a user on the basis of its channel quality and frame delay. This packet scheduling algorithm is then combined with a buffer management strategy that discards packet and other dependent packets if they are likely to arrive at the user end after the playback deadline. The playback deadline is the time when a frame is needed for video playback and each frame is attributed with this deadline. A packet scheduling solution that aimed to improve the perceptual quality among all video users was developed in [12]. The developed packet scheduling algorithm schedules packets of a user according to its perceptual quality, channel quality and decoding deadline. Decoding deadline is the time when a frame is needed for decoding at a user end. The buffer management strategy developed in this study discards a packet and other dependent packets at base station if the time when the packet is needed for decoding at a user end has passed. Similarly, the authors in [13] developed a solution that attempts to improve perceptual quality across all video users. The packet scheduling algorithm developed in the study determines priority of a user's packets according to its channel quality, average throughput, priority of a frame, playback buffer information (the playback buffer is located at Application Layer at a user end) and whether any packets have been transmitted to the user or not. If a packet is discarded at a user for playback deadline violation, the developed buffer management strategy discards all packets that are dependent to the discarded packet at the base station. 
The aforementioned studies mostly focus in satisfying either the perceptual quality or video playback continuity, but not both. A continuous video playback does not mean that an excellent perceptual quality is experienced at the user (i.e. the video playback may be continuous but with a poor perceptual quality as a number of frames in a video stream is lost). Similarly, a user may experience a good perceptual quality but has to tolerate with a large number of playback interruptions (i.e. a video playback is interrupted if a frame is not available when it is needed for playback or due to playback buffer underflow). To address this situation, this paper proposes a novel packet scheduling solution known as Opportunistic and Playback-Sensitive Scheduling (OPSS) for usage in the downlink LTE. OPSS is a combination of a buffer management strategy and a packet scheduling algorithm. It aims to optimize the system capacity (number of users) without compromising the QoS constraint of video streaming (i.e. a continuous video playback at the highest perceptual quality for each user).

The remaining sections of this paper are organized as follows: Section 2 gives an overview of the video streaming followed by a detailed description of the proposed packet scheduling solution in Section 3. Section 4 contains environment of the simulation while Section 5 evaluates performance of the OPSS against a well-known packet scheduling algorithm. Finally, Section 6 concludes the paper.

\section{VIDEO STREAMING OVERVIEW}

As previously discussed in Section 1, simultaneous streaming of multiple video sessions requires a continuous video playback at the highest perceptual quality at each user. Generally, the perceptual quality is measured on the basis of Peak-Signal-to-Noise-Ratio (PSNR) [14] or Mean Opinion Score (MOS) [15] metrics. Note that the PSNR of a user can be mapped to a MOS value to give a qualitative representation of the perceptual quality. The MOS contains a scaled value from 1 to 5 that implies a bad to an excellent perceptual quality (as illustrated in Table 1).

Table 1. Average PSNR to MOS mapping [16]

\begin{tabular}{|c|c|c|}
\hline $\begin{array}{c}\text { Lowest } \\
\text { Average } \\
\text { PSNR (dB) }\end{array}$ & $\begin{array}{c}\text { Minimum } \\
\text { MOS }\end{array}$ & $\begin{array}{c}\text { Perceptual } \\
\text { Quality }\end{array}$ \\
\hline$<20$ & 1 & Bad \\
\hline $20-25$ & 2 & Poor \\
\hline $25-31$ & 3 & Fair \\
\hline $31-37$ & 4 & Good \\
\hline$>37$ & 5 & Excellent \\
\hline
\end{tabular}

Video streaming allows a user to start a video playback without receiving an entire video stream. A typical approach for ensuring a continuous playback is to delay the start of a video playback until the total number of frames in the playback buffer exceeds a buffering threshold [17]. The buffering threshold is defined as the maximum number of frames required to be filled in the playback buffer at a user end before the user can start or resume a video playback.

The video streaming properties and the mobile cellular channels characteristics may lead to interruptions during the video playback $[18,19]$. Interruption occurs when the total number of frames in the playback buffer is less than a playback buffer underflow threshold. The playback buffer underflow threshold is defined as the maximum number of frames required to be available 
in the playback buffer for a continuous playback. The video playback resumes at the same position where the interruption occurs after the total number of frames in the playback buffer exceeds the specified buffering threshold.

A user will not be satisfied if it has to wait longer to start or resume its video playback. Freezing Delay Ratio (FDR) metric [6] which gives a total delay experienced by each user throughout its video session is used to measure the video playback continuity. Note that a low FDR implies that all users are likely to experience continuous video playback throughout their sessions. A high FDR indicates that a number of users are likely to have waited for an amount of time before they can start their video playback or there a number of interruptions occurred throughout these users' video sessions.

An example of the freezing delay where it takes $\varepsilon \mathrm{ms}$ for the first bit of the video stream to arrive and being stored in the playback buffer after a user requests for a video session is illustrated in Figure 1. The video playback starts after it has been delayed for $\alpha \mathrm{ms}$. This delay is used to fill in the playback buffer such that the total number of frames in the playback buffer exceeds the buffering threshold. The video playback is interrupted if the total number of frames is less than the playback buffer underflow threshold. The video playback resumes after $\beta \mathrm{ms}$. This time duration is used to re-fill in the playback buffer such that the total number of frames in the playback buffer exceeds the buffering threshold.

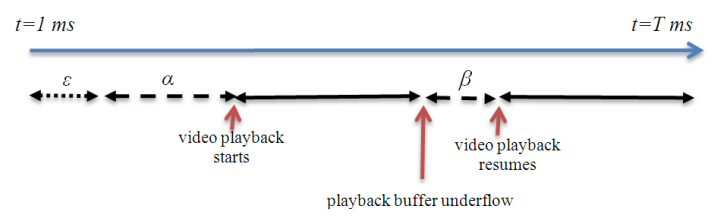

Figure 1. Example of freezing delay during a video session

A frame needs to be decoded first before it can be played back. Some frames cannot be decoded if a higher priority frame is lost. These frames are discarded at the user. A number of studies (i.e. [7, $11,12])$ considered a scenario that discards a frame and other dependent frames if the frame arrives at the playback buffer after its decoding or playback deadline. These studies generally aimed to minimize frame loss ratio as the loss of frames due to frame discards degrades the perceptual quality experienced at the users. However, this paper considers a scenario that does not discard packets/frames for decoding/playback deadline violation.

\section{Opportunistic and Playback Sensitive Scheduling}

This section presents a novel packet scheduling solution called OPSS so as to ensure a continuous video playback at the highest perceptual quality for more users. This solution is proposed for usage in the multi-carrier downlink LTE. Detailed descriptions of the buffer management strategy and packet scheduling algorithm of the OPSS are discussed in the following sections.

\subsection{Buffer Management Strategy}

All packets arriving at the user end are delivered in sequence towards the playback buffer through the use of re-sequencing buffer. Whenever a TB (with a known Transmission Sequence Number TSN) is erroneously received at the user, all subsequent TBs with higher TSNs that are correctly received by the user are stored in the re-sequencing buffer and a re-sequencing timer is started. The packets of out-of-sequence TBs are delivered towards the playback buffer upon a correct reception of the erroneous TB or upon expiry of the re-sequencing timer [20]. In this paper, 
packets of a TB are discarded and considered as lost packets only if the TB has exceeded maximum number of retransmissions or the re-sequencing timer associated with the TB has expired.

The loss of video packets will degrade video quality. Degradation will be significant if higher priority packets that are to be used for decoding other dependent packets are lost. Whenever a packet is lost, there may be one or more packets belonging to the same frame to the lost packet or are dependent to the lost packet are still residing in the base station buffer. These packets should not be transmitted to the user as the packets cannot be used for decoding or video playback (due to their dependency to the lost packet). Therefore, the buffer management strategy of the OPSS discards these packets from the base station buffer such that the available radio resources are efficiently used for (re)transmission of packets that can be used for decoding and video playback. All packets are stored in a transmission buffer at the base station upon transmission. The time duration that each packet has been residing in the transmission buffer is known to the base station. Moreover, the maximum duration that a packet can reside in a re-sequencing buffer at the user is also known at the base station. One or more packets may have resided long enough within the transmission buffer such that, if they are retransmitted, they are likely to arrive at the user end after the re-sequencing timer expires. To avoid this situation, the buffer management strategy discards these packets from the transmission buffer.

The buffer management strategy proposed in this paper is almost similar to the strategies discussed in [7, 11-13] that discard packets at the base station (i) due to packets dependency or (ii) if the packets are likely to arrive at the user after the deadline. Additionally, similar to [13], the playback buffer information is considered in the proposed packet scheduling algorithm as it plays a role in ensuring a continuous video playback [19]. Note that the playback buffer is only updated when all packets belonging to a frame have correctly arrived at the playback buffer. On the contrary, the OPSS differs from other solutions as the proposed packet scheduling algorithm uses different formulation when determining priority of each user for packets transmission. Detailed description of the proposed packet scheduling algorithm is described next.

\subsection{Packet Scheduling Algorithm}

In each scheduling interval and on each $\mathrm{RB}$, the packet scheduling algorithm of the OPSS schedules a user that maximizes $\mu_{i, j}(t)$ in the following equation:

$$
\begin{aligned}
& \mu_{i, j}(t)=\left(\frac{r_{i, j}(t)}{\left(R_{i}(t)\right)^{2}}\right) *\left(1-\frac{P B_{i}(t)}{\alpha_{p b}}\right) \\
& R_{i}(t+1)=\left(1-\frac{1}{t_{c}}\right) R_{i}(t)+\frac{1}{t_{c}} * r_{c} t_{i}(t+1) \\
& \operatorname{rtot}_{i}(t+1)=\sum_{j=1}^{R B_{\max }} r_{i, j}(t+1)
\end{aligned}
$$

where $\mu_{i, j}(t)$ is the priority of user $i$ on RB $j$ at scheduling interval $t, r_{i, j}(t)$ is the instantaneous data rate of user $i$ on RB $j$ at scheduling interval $t, R_{i}(t)$ is the average throughput of user $i$ at scheduling interval $t, P B_{i}(t)$ is the total number of frames in playback buffer of user $i$ at scheduling interval $t, \alpha_{p b}$ is the playback buffer weighting factor (it scales the second multiplicand in Equation (1) to be between 0 and 1), $t_{c}$ is a time constant, $r t o t_{i}(t+1)$ is the total data rate being used to transmit packets to user $i$ at scheduling interval $t+1$, and $R B_{\max }$ is the maximum available number of RBs. Note that the playback buffer weighting factor is used to avoid the scheduling decision to be dependent upon playback buffer information only as the value for the first multiplicand in Equation (1) is between 0 and 1. 
The proposed algorithm is more likely to give scheduling opportunity to a user with the least number of frames in its playback buffer if the channel quality and the average throughput of each user are similar. If one or more TBs are erroneously received at the user, it will take a longer time to update the playback buffer as all subsequent TBs with higher TSNs than the erroneous TBs that may have been correctly arrived at the user are stored in the re-sequencing buffer. The packets of out-of-sequence TBs are only delivered to the playback buffer upon correct reception of the erroneous TBs or upon expiry of the re-sequencing timer. Scheduling opportunity is highly likely to be given to this user in subsequent scheduling intervals if its playback buffer is not updated. The average throughput is used in the proposed algorithm to compensate for the effect of the delay in playback buffer update. In this case, the user priority decreases in subsequent scheduling intervals as more packets are transmitted to the user. After a number of scheduling intervals, even if the user has the least number of frames in its playback buffer, scheduling opportunity is highly likely to be given to other users due to the transmission history of this user.

It can be observed in Equation (1) that the formulation of the proposed packet scheduling algorithm is dependent upon three variables namely channel quality, average throughput and playback buffer information. The relevance of each variable is justified next. A number of studies (i.e. [21-24]) have shown that scheduling opportunity that considers channel quality can significantly improve the system performance. Therefore, this variable is considered in the proposed algorithm. However, even though the packets of each selected user can be transmitted with a better modulation and coding scheme, the packet scheduling algorithm that is dependent upon channel quality alone is inefficient in fairness as it deprives users located at the cell edge from receiving their packets.

To address the fairness problem, the average throughput variable is taken into consideration in the proposed algorithm. The update of the average throughput increases the priority of users whose packets are not scheduled in the previous scheduling intervals. Therefore, the packets of these users are likely to be scheduled in the sub-sequent scheduling intervals. Additionally, this variable is used to compensate for the effect of delay in playback buffer update (as discussed previously in this section). Finally, the playback buffer information is considered in order to ensure that all users are given an equal opportunity for video playback. This allows the OPSS to minimize the delay before the users can start or resume their video playback. Moreover, this variable attempts to maintain a continuous video playback by giving a higher priority for packets transmission to the users with least number of frames in their playback buffer (if the channel quality and the average throughput of each user are similar) so as to avoid the video playback from being interrupted due to playback buffer underflow.

\section{Simulation ENVIRONMENT}

The performance of the OPSS was evaluated within a single hexagonal cell scenario of $5 \mathrm{MHz}$ bandwidth with $25 \mathrm{RBs}$ and $2 \mathrm{GHz}$ carrier frequency. The base station has a fixed location at the centre cell and it was assumed that equal transmit power $(43.01 \mathrm{dBm}$ total base station transmit power) is used on each RB. Each user moves at a constant speed of $3 \mathrm{~km} / \mathrm{h}$. These users are uniformly located within the cell. The Cost-231 HATA model for an urban environment [25], a Gaussian log-normal distribution with 0 mean and $8 \mathrm{~dB}$ standard deviation [26] and a frequencyflat Rayleigh fading model [27] are used to model the radio propagation channel. The probability that the channel quality information report is in error was fixed at $1 \%$ and this report is only available for use by the base station after a 4 ms delay [28]. It was assumed that besides the CQI and Hybrid Automatic Repeat Request (HARQ) information, the feedback from a user also contains playback buffer information. 
Three video streams which were downloaded from a publicly available video traces [4] were used. The video streams were encoded with a frame rate of $30 \mathrm{fps}$ (frames per second). One GoP contains 16 frames with IBBBPBBBPBBBPBBB sequence. Each frame has a number, type (I, P or B frame), decoding/playback deadline, size and PSNR value of the luminance component. A user randomly requests one video stream throughout its session and the request arrives at the base station at the beginning of the simulation. It was assumed in this performance study that: (i) a video playback is interrupted if a frame is not available when it is needed for playback (i.e. the frame is not discarded if it arrives at the playback buffer after the playback deadline $[18,19]$ ), (ii) the playback buffer capacity of each user is infinite and (iii) the playback buffer underflow threshold is fixed at 5 frames.

Minimum MOS and FDR metrics are used for performance evaluation. In this paper, the minimum MOS evaluates the perceptual quality where a user with the lowest average PSNR among other video users is considered. In this case, a low minimum MOS (i.e. minimum MOS $=1$, see Table 1) indicates that at least one user is experiencing a bad perceptual quality whereas a high minimum MOS (i.e. minimum MOS=4) indicates that all users are experiencing at least a good perceptual quality. The FDR is defined in this paper as the average of the ratio of total freezing delay of each user to the total simulation time. Equation (4) gives the mathematical expression of the FDR [29]:

$$
F D R=\frac{1}{N} \sum_{i=1}^{i=N} \frac{D f_{i}}{T}
$$

where $D f_{i}$ is the total freezing delay of user $i, T$ is the total simulation time and $N$ is the total number of users.

The results obtained via computer simulation of the OPSS solution were evaluated and compared with the well-known PF algorithm. The PF was chosen as it is one of the packet scheduling algorithms that provides considerably good performance in the legacy single-carrier mobile cellular systems. Moreover, due to its efficiency, the authors in [30-33] all extended the PF algorithm into multi-carrier mobile cellular systems. Packet scheduling in the single-carrier mobile cellular systems allocates all of the available radio resources to a single user in each scheduling interval. As such, the PF equations that support packet scheduling in the downlink LTE system as described [33] is used in this performance evaluation.

\section{Performance Results and Discussions}

The following sections compare the performance of the OPSS with the PF algorithm for different system capacities and buffering threshold.

\subsection{Performance Comparison with Increasing System Capacity}

Figure 2 and Figure 3 show the minimum MOS and FDR of the OPSS solution and PF algorithm with increasing system capacity. The buffering threshold of each user was fixed at $200 \mathrm{~ms}$ in this performance comparison. It can be observed that the minimum MOS and FDR degrade with increasing system capacity as there are insufficient RBs to schedule the video packets from the users. Table 2 shows that if at least a good perceptual quality has to be satisfied for all users, then the maximum system capacities that the OPSS and PF can simultaneously support are 35 and 25 users respectively. This is equivalent to $40 \%$ improvement in the system capacity achieved in the OPSS over the PF algorithm. Moreover, it can be observed in Table 3 that when the system capacity is at 35 users, the OPSS significantly minimizes the FDR by $42.6 \%$ compared to the PF 
International Journal of Wireless \& Mobile Networks (IJWMN) Vol. 6, No. 1, February 2014

algorithm. This indicates that the OPSS solution is superior to the PF algorithm in providing a continuous playback for all video users.

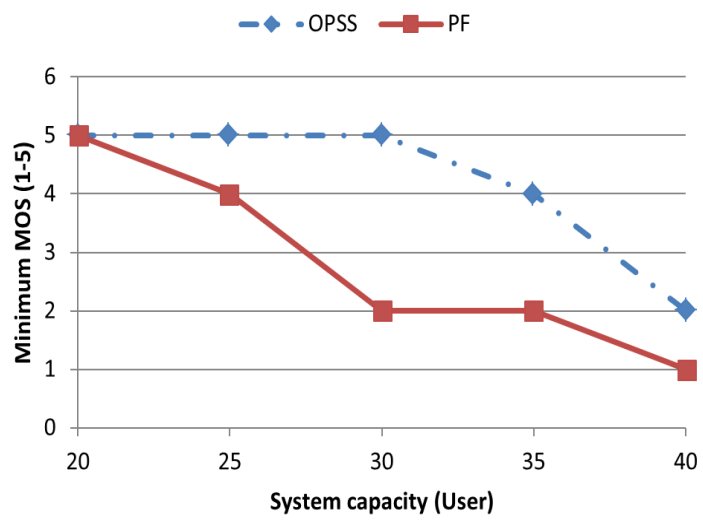

Figure 2. Minimum MOS vs. system capacity

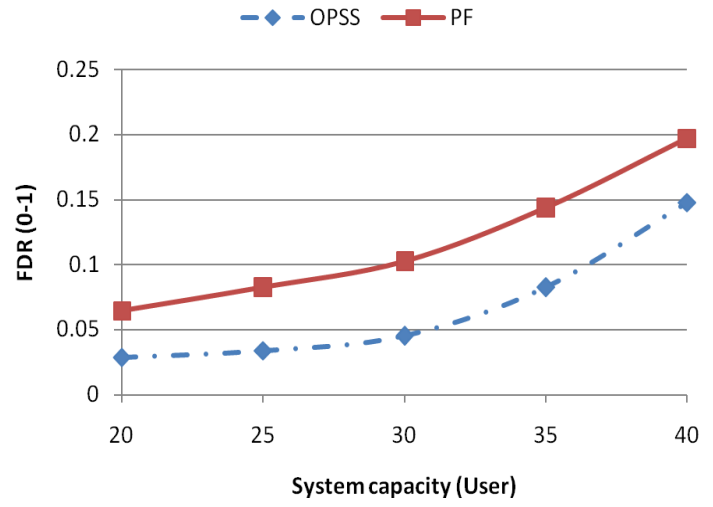

Figure 3. FDR vs. system capacity

Table 2. Maximum system capacities to support a range of minimum MOS

\begin{tabular}{|c|c|c|c|}
\hline \multirow{2}{*}{$\begin{array}{c}\text { Minimum } \\
\text { MOS }\end{array}$} & \multirow{2}{*}{$\begin{array}{c}\text { Perceptual } \\
\text { Quality }\end{array}$} & \multicolumn{2}{|c|}{$\begin{array}{c}\text { Maximum } \\
\text { System capacity }\end{array}$} \\
\cline { 3 - 4 } & & OPSS & PF \\
\hline 1 & Bad & $>40$ & 40 \\
\hline 2 & Poor & 40 & 30 \\
\hline 3 & Fair & 37.5 & 27.5 \\
\hline 4 & Good & 35 & 25 \\
\hline 5 & Excellent & 20 & 20 \\
\hline
\end{tabular}


International Journal of Wireless \& Mobile Networks (IJWMN) Vol. 6, No. 1, February 2014

Table 3. FDR at 35 users

\begin{tabular}{|c|c|c|}
\hline & FDR & $\begin{array}{c}\text { Improvement in } \\
\text { OPSS over PF (\%) }\end{array}$ \\
\hline PF & 0.143571 & 42.6 \\
\hline OPSS & 0.082446 & 42.6 \\
\hline
\end{tabular}

The improvements in the minimum MOS and FDR performances in OPSS compared to the PF algorithm can be attributed to the following factors. The buffer management strategy enables the OPSS to efficiently utilize the available RBs by selectively (re)transmitting packets that can be used for decoding and video playback. Furthermore, OPSS integrates the playback buffer information in the packet algorithm so as to ensure a continuous video playback for each user.

\subsection{Impact of Buffering Threshold on Performance}

The impact of buffering threshold on the OPSS solution and PF algorithm are studied in this section. In this performance comparison, the system capacity was fixed at 25 users (i.e. the system capacity where all users experience at least a good perceptual quality in OPSS and PF - as shown in Figure. 2). As previously discussed in Section 2, the start or resume of a video playback is delayed with increasing buffering threshold because more frames need to be filled in the playback buffer. As there is insufficient time to playback all of the video frames of a user (i.e. the maximum duration of a video session is fixed at $T \mathrm{~ms}$ for each user), one or more frames of the user may not be able to be played back at the end of its session and this leads to degradations of the minimum MOS and FDR with increasing buffering threshold (as shown in Figure 4 and Figure 5).

Figure 4 shows that the OPSS minimizes degradation due to the impact of the buffering threshold because it is capable of providing an excellent perceptual quality (minimum MOS=5) for all users for up to $600 \mathrm{~ms}$ buffering threshold. On the other hand, at least one user in the PF algorithm experienced a bad perceptual quality (minimum MOS $=1$ ) at the $600 \mathrm{~ms}$ buffering threshold. This implies that the majority of the frames of a number of users cannot be played back when their video sessions end due to the time taken to start or resume the video playback.

When compared with the PF algorithm, it can be observed in Figure 5 that the OPSS significantly minimizes the FDR at a lower buffering threshold. The packet scheduling algorithm of the OPSS gives a higher priority for a user with the least number frames to receive its packets. At a lower buffering threshold, the users in the OPSS can fill their playback buffer with sufficient number of frames earlier than the PF algorithm and hence allowing OPSS to minimize the delay to start or resume the video playback. Table 4 shows that the OPSS minimizes the FDR by $58.7 \%$ compared to the PF algorithm at $200 \mathrm{~ms}$ buffering threshold. 
International Journal of Wireless \& Mobile Networks (IJWMN) Vol. 6, No. 1, February 2014

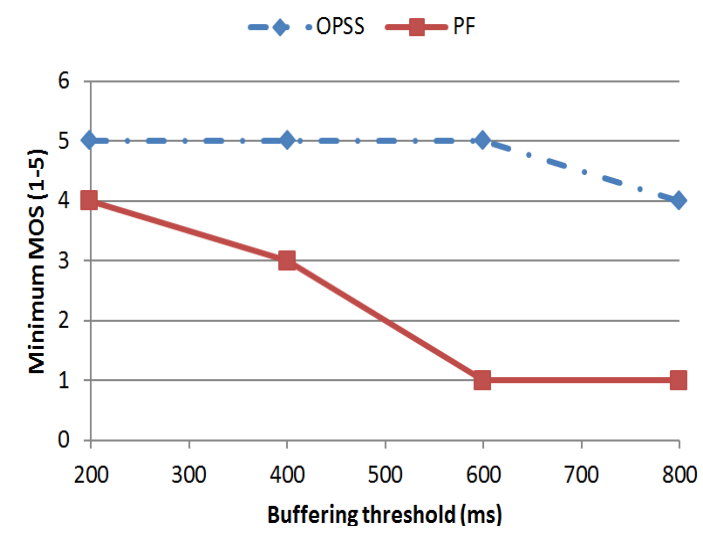

Figure 4. Minimum MOS vs. buffering threshold

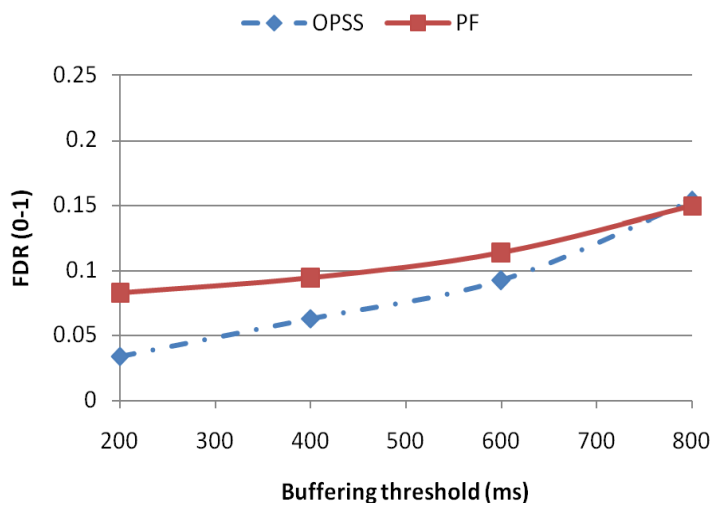

Figure 5. FDR vs. buffering threshold

The PF algorithm is likely to outperform the OPSS in terms minimizing the FDR when the buffering threshold is over $800 \mathrm{~ms}$. This is because there is only a limited number of frames that can be played back in the PF algorithm (i.e. minimum MOS=1 in the PF at $800 \mathrm{~ms}$ buffering threshold). Note that the freezing delay is only computed for the frames that can be played back at the users. On the other hand, even if the FDR in the OPSS is likely to be worse than the PF algorithm when the buffering threshold increases above $800 \mathrm{~ms}$, the OPSS guarantees that majority of the frames can be played back throughout each user's session and hence improving the perceptual quality experienced at the users.

Table 4. FDR at $200 \mathrm{~ms}$ buffering threshold

\begin{tabular}{|c|c|c|}
\hline & FDR & $\begin{array}{c}\text { Improvement in } \\
\text { OPSS over PF (\%) }\end{array}$ \\
\hline PF & 0.082552 & 58.7 \\
\hline OPSS & 0.034056 & \\
\hline
\end{tabular}


International Journal of Wireless \& Mobile Networks (IJWMN) Vol. 6, No. 1, February 2014

\section{CONCLuSiON}

Simultaneous streaming of multiple video sessions is a challenging task due to the QoS constraint of video streaming as well as unreliable and resource-constrained of the downlink LTE. A number of studies that attempted to address this challenge by improving the perceptual quality or ensuring video playback continuity have been developed. This paper proposes a novel solution that combines the buffer management strategy with packet scheduling algorithm to improve video streaming performance in the downlink LTE. It was demonstrated via computer simulation that the proposed solution is particularly effective in providing a continuous video playback with good perceptual quality for more users. If at least a good perceptual quality is to be satisfied for all users (QoS constraint of video streaming), then the proposed solution improves the system capacity by $40 \%$ over the PF algorithm. Moreover, it minimizes the video playback interruption by $42.6 \%$ as compared to the PF algorithm. The proposed solution has a low computational complexity and hence suited for implementation in the downlink LTE and other multi-carrier mobile cellular systems without additional hardware cost.

\section{ACKNOWLEDGMENT}

This work is supported by International Islamic University Malaysia (IIUM) Endowment Fund Type B (EDW B11-198-0676).

\section{REFERENCES}

[1] Holma, H. \& Toskala, A. (2009) LTE for UMTS: OFDMA and SC-FDMA Based Radio Access. John Wiley \& Sons Ltd.

[2] Fan, L., Guizhong, L. \& Lijun, H. (2009) Application-Driven Cross-Layer Approaches to Video Transmission over Downlink OFDMA Networks. in IEEE Global Telecommunications Workshops, 1-6.

[3] Sun, H. Vetro, A. \& Xin, J. (2007)An Overview of Scalable Video Streaming. Wireless Communications and Mobile Computing, 7(2), 159-172.

[4] Seeling, P., Reisslein, M. \& Kulapala, B. (2004) Network Performance Evaluation Using Frame Size and Quality Traces of Single-Layer and Two-Layer Video: A Tutorial. IEEE Communications Surveys \& Tutorials, 6(3), 58-78.

[5] Haghani, E. Shyam, P. Doru, C. Eunyoung, K. \& Ansari, N. (2009) A Quality-Driven Cross-Layer Solution for MPEG Video Streaming Over WiMAX Networks. IEEE Transactions on Multimedia, 11(6), 1140-1147.

[6] Ozcelebi, T., Sunay, M.O., Tekalp, A.M. \& Civanlar, M.R. (2005) Cross-Layer Design for Real-Time Video Streaming over 1xEV-DO using Multiple Objective Optimization, in IEEE Global Telecommunications Conference, 2761-2766.

[7] Haghani, E., Ansari, N., Shyam, P. \& Doru, C. (2010) Traffic-Aware Video Streaming in Broadband Wireless Networks. in IEEE Wireless Communications and Networking Conference, 1-6.

[8] Yongjin, C. Kuo, C.C.J., Renxian, H. \& Lima, C. (2009) Cross-Layer Design for Wireless Video Streaming. in IEEE Global Telecommunications Conference, 1-5.

[9] Jalali, A., Padovani, R. \& Pankaj, R. (2000) Data Throughput of CDMA-HDR a High EfficiencyHigh Data Rate Personal Communication Wireless System. in IEEE 51st Vehicular Technology Conference Proceedings, 1854-1858. 
International Journal of Wireless \& Mobile Networks (IJWMN) Vol. 6, No. 1, February 2014

[10] Boggia, G., Camarda, P., Fortuna, R. \& Grieco, L.A. (2009) A Scheduling Strategy to Avoid Playout Interruptions in Video Streaming Systems. in Proceedings of 18th Internatonal Conference on Computer Communications and Networks. 1-6.

[11] Junhua, T., Liren, Z. \& Chee-Kheong, S. (2006) An Opportunistic Scheduling Algorithm for MPEG Video Over Shared Wireless Downlink. in IEEE International Conference on Communications. 872877.

[12] Honghai, Z., Yanyan, Z., Khojastepour, M.A. \& Rangarajan, S. (2009) Scalable Video Streaming over Fading Wireless Channels. in IEEE Wireless Communications and Networking Conference. 1-6.

[13] Tupelly, R.S., Zhang, J. \& Chong, E.K.P. (2003) Opportunistic Scheduling for Streaming Video in Wireless Networks. in Conference on Information Sciences and Systems. 1-6.

[14] Seeling, P., Reisslein, M. \& Fitzek, F.H.P. (2006) Layered Video Coding Offset Distortion Traces for Trace-Based Evaluation of Video Quality after Network Transport. in 3rd IEEE Consumer Communications and Networking Conference, 292-296.

[15] Zhenzhong, C., Maodong, L. \& Yap-Peng, T. (2010) Perception-Aware Multiple Scalable Video Streaming Over WLANs. IEEE Signal Processing Letter. 17(7), 675-678.

[16] Klaue, J., Rathke, B. \& Wolisz, A. (2003) Evalvid - A Framework for Video Transmission and Quality Evaluation. in 13th International Conference on Modelling Techniques and Tools for Performance Evaluation. 1-5.

[17] Guanfeng, L. \& Ben, L. (2008) Effect of Delay and Buffering on Jitter-Free Streaming Over Random VBR Channels. IEEE Transactions on Multimedia, 10(6), 1128-1141.

[18] Vukadinovic, V. \& Karlsson, G. (2010) Video Streaming Performance under Proportional Fair Scheduling. IEEE Journal on Selected Areas in Communications, 28(3),399-408.

[19] Ozcelebi, T., Oguz, S.M., Murat, T.A. \& Reha, C.M. (2007) Cross-Layer Optimized Rate Adaptation and Scheduling for Multiple-User Wireless Video Streaming. IEEE Journal on Selected Areas in Communications, 25(4), 760-769.

[20] Larmo, A., Lindstrom, M., Meyer, M., Pelletier, G., Torsner, J. \& Wiemann, H. (2009) LTE LinkLayer Design. IEEE Communications Magazine, 47(4), 52-59.

[21] Andrews, M., Kumaran, K., Ramanan, K., Stolyar, A., Whiting, P. \& Vijayakumar, R. (2001) Providing Quality of Service over a Shared Wireless Link. IEEE Communications Magazine, 39(2), 150-154.

[22] Tsybakov, B.S. (2002) File Transmission over Wireless Fast Fading Downlink. IEEE Transactions on Information Theory, 48(8), 2323-2337.

[23] Ramli, H. A. M. \& Sandrasegaran, K. (2013) Robust Scheduling Algorithm for Guaranteed Bit Rate Services. International Journal of Mobile Communications, 11(1), 71-88.

[24] Pokhariyal, A., Monghal, G., Pedersen, K.i., Mogensen, P.E., Kovacs, I.Z., Rosa, C. \& Kolding, T.E. (2007) Frequency Domain Packet Scheduling Under Fractional Load for the UTRAN LTE Downlink. in IEEE Vehicular Technology Conference, 699-703.

[25] Rappaport, T.S. (2003) Wireless Communications: Principles and Practice. Prentice Hall.

[26] Gudmundson, M. (1991) Correlation Model for Shadow Fading in Mobile Radio Systems. Electronics Letters, 2145-2146.

[27] Komninakis, C. (2003) A Fast and Accurate Rayleigh Fading Simulator. in IEEE Globecom, 33063310 .

[28] Pedersen, K.I., Monghal, G., Kovacs, I.Z., Kolding, T.E., Pokhariyal, A., Frederiksen, F. \& Mogensen, P. (2007) Frequency Domain Scheduling for OFDMA with Limited and Noisy Channel Feedback. in Vehicular Technology Conference, 1792-1796.

[29] Ramli, H.A.M. Sandrasegaran, K., Basukala, R., Patachaianand, R. \& Afrin, T.S (2011) Video Streaming Performance under Well-Known Packet Scheduling Algorithms. International Journal of Wireless \& Mobile Networks (IJWMN), 3(1), 25-38. 
[30] Ruangchaijatupon, N. \& Ji, Y. (2008) Simple Proportional Fairness Scheduling for OFDMA FrameBased Wireless Systems. in IEEE Wireless Communications and Networking Conference, 1593-1597.

[31] Mugen, P. \& Wenbo, W. (2004) Advanced HARQ and Scheduler Schemes in TDD-CDMA HSDPA Systems. in Joint Conference of the 10th Asia-Pacific Conference on Communications and the 5th International Symposium on Multi-Dimensional Mobile Communications, 67-70.

[32] Wang, Y. \& Yang, H. (2003) Retransmission Priority Scheduling Algorithm for Forward Link Packet Data Service. in International Conference on Communication Technology Proceedings, 926-930.

[33] Ramli, H.A.M., Basukala, R., Sandrasegaran, K. \& Patachaianand, R. (2009) Performance of Well Known Packet Scheduling Algorithms in the Downlink 3GPP LTE System. in IEEE 9th Malaysia International Conference on Communications (MICC), 815-820.

\section{Authors}

Huda Adibah Mohd Ramli received her $\mathrm{PhD}$ in Engineering from University of Technology, Sydney (Australia) (2012), MSc in Software Engineering from the University of Technology Malaysia (Malaysia) (2006) and BEng in Electrical and Computer Engineering from International Islamic University Malaysia (Malaysia) (2003). Her current research interest generally focuses on radio resource management in the mobile communication systems specifically on packet scheduling and component carrier selection

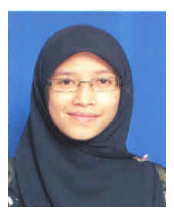

Kumbesan Sandrasegaran holds a PhD in Electrical Engineering from McGill University (Canada) (1994), a Masters of Science Degree in Telecommunication Engineering from Essex University (UK) (1988) and a Bachelor of Science (Honours) Degree in Electrical Engineering (First Class) (UZ) (1985). He was a recipient of the Canadian Commonwealth Fellowship (1990-1994) and British Council Scholarship (1987-1988). He is a Professional

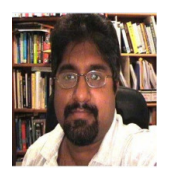
Engineer (Pr.Eng) and has more than 20 years experience working either as a practitioner, researcher, consultant and educator in telecommunication networks. During this time, he has focused on the planning, modeling, simulation, optimisation, security, and management of telecommunication networks 